\title{
Perceptual capacity limits in visual detection and search
}

\author{
WILLIAM PRINZMETAL \\ Princeton University, Princeton, New Jersey \\ and \\ WILLIAM P. BANKS \\ Pomona College, Claremont, California
}

\begin{abstract}
The present research tests limited- and unlimited-capacity theories of perception by comparing accuracy in detection and search tasks under conditions of simultaneous and successive presentation of array items. The unlimited-capacity approach predicts no difference between simultaneous and successive presentation when nonperceptual factors are controlled. The limitedcapacity approach predicts an advantage for successive presentation when the number of simultaneously presented items exceeds processing capacity. In five experiments using a consistent mapping paradigm, successive presentation was reliably better than simultaneous presentation.
\end{abstract}

The present study tests limited- and unlimited-capacity theories of visual perception by comparing simultaneous and successive presentation of array elements in detection and search tasks. The two theoretical positions have different predictions for simultaneous and successive presentation. The unlimited-capacity approach assumes that limitations in the detection of a target item in an array of noise items can be traced either to sensory or to decision factors. If these are adequately controlled, there should be no difference in performance when all the stimulus items are presented simultaneously and when they are presented one at a time (successive presentation). The limited-capacity view is that there is a perceptual limitation on the amount of information that can be processed or extracted from the stimulus in a given interval of time, independent of sensory or decision processes, and it predicts an advantage for successive presentation when all other factors are controlled for (Duncan, 1980a).

A number of early studies manipulated presentation rate or compared simultaneous and successive presentations and found no effect on performance, thus supporting the unlimited-capacity view (Eriksen \& Spencer, 1969; Shiffrin \& Gardner, 1972). These experiments

This research was supported by National Science Foundation Grant BNS 78-17442 and NIH Grant 1 R01 MH33279-01, both to W. P. Banks. The research is based on the PhD dissertation, submitted to Claremont Graduate School, of the first author. We wish to thank Deborah Burke and Robin Mermelstein for their helpful comments on this paper and Antonio Estrada, Michele Matule, and Grayson Barber for help in performing the experiments. Reprint requests should be sent to William Prinzmetal, Department of Psychology, Princeton University, Princeton, New Jersey 08544. have been cited widely as evidence for unlimited-capacity theories (e.g., Schneider \& Shiffrin, 1977; Spoehr \& Lehmkuhle, 1982). However, several, more recent studies have found evidence for capacity limitations when this paradigm was used. Hoffman (1978, 1979), using a varied mapping (VM) procedure, presented stimulus letters successively with onset asynchronies of from 0 (simultaneous presentation) to $100 \mathrm{msec}$. In a varied mapping procedure, items that are noise elements on some trials are targets on other trials. Detection performance was better with successive than with simultaneous presentation when the display size was eight letters, but not when the display size was four letters (Hoffman, 1979).

Duncan (1980b), using a consistent mapping (CM) paradigm, also found an advantage for successive over simultaneous presentation. In a CM paradigm, stimuli that are targets never serve as distractors. Duncan (1980b) presented four items either all at once or a pair of items at a time. Target items could appear independently with a probability of $1 / 3$ in either of the pairs. Subjects were to respond "yes" if there was a target in either pair of items or in both pairs. Performance was measured with $d^{\prime}$. Unfortunately, this experiment cannot be taken as unequivocal evidence for capacity limits in a CM paradigm. Shaw (1982) showed that when subjects must make a yes-no response based on information from two independent sources, $d^{\prime}$ may not reflect changes in sensitivity, but only changes in subjects' criteria.

The purpose of the experiments reported here was to see if we could obtain an advantage for successive presentation in a CM paradigm with both search and detection tasks. In addition, we investigated the effects of practice, lateral masking, target-noise confusability, 
noise homogeneity, and target onset order on performance with simultaneous and successive presentation.

\section{EXPERIMENT 1}

The first experiment was a two-alternative forcedchoice task in which nine display items were presented either simultaneously or successively. To test whether capacity limitations could be overcome with practice in a $\mathrm{CM}$ paradigm, the difference between simultaneous and successive presentation was compared in the first and second halves of the experiment. The experiment also compared simultaneous presentation with two rates of successive presentation. Finally, the experiment compared homogeneous and heterogeneous noise to test whether the lack of an improvement from simultaneous to successive presentation in some previous studies resulted from the use of homogeneous noise. (see Experiment 3 of Hoffman, 1979).

\section{Method}

Procedure. The subjects were briefly shown arrays of nine letters arranged in a $3 \times 3$ matrix and had to decide on each trial whether the arrays contained an $F$ or a $T$. The arrays always contained eight noise elements that were either all the same letter or were eight different letters. The noise letters were E, J, K, L, P, R, U, and Z. Array items were presented either simultaneously or successively. On successive presentation, the interstimulus interval (ISI) was $20 \mathrm{msec}$ for half the subjects and $70 \mathrm{msec}$ for the other half.

The stimuli appeared on a cathode-ray tube (CRT) with a P40 dual phosphor and were viewed through a blue filter (Kodak Wratten No. 47) to render invisible the long-persistence yellow phosphor trace. The subjects saw the display at a distance of $57 \mathrm{~cm}$. All stimulus letters subtended a visual angle of approximately .4 deg of visual angle vertically and $.3 \mathrm{deg}$ of visual angle horizontally. Stimulus items were spaced $1 \mathrm{deg}$ apart, center to center.

Each trial began with a fixation dot that appeared in the center of the field and that remained on for $500 \mathrm{msec}$. The dot was replaced by the stimulus array, in which the letters were presented either simultaneously or successively. The exposure duration was approximately $67 \mathrm{msec}$ for the entire simultaneous arrays and the same duration for each successive letter for successive presentations (five computer refreshes on the CRT at $60 \mathrm{~Hz}$ ). The stimulus letters were followed again by the fixation dot for $500 \mathrm{msec}$, and then the field became blank. The subjects were instructed to respond after the field became blank by pressing one of two microswitches to indicate which target had been presented. The subjects were instructed to respond as accurately as possible.

The target letter could appear in any of eight positions (the center position was excluded), and there were eight different noise elements, thus, there were 128 trials per block ( 2 targets $\mathrm{x}$ 8 positions $\times 8$ noise letters). In successive presentation, items were presented in a random order. (Item order was not a factor in the design.) The four between-block conditions were homogeneous vs. heterogeneous noise crossed with simultaneous vs. successive presentation. Each subject received each betweenblock condition twice in a different counterbalanced order, and each of these conditions occurred equally often in each ordinal position, for a total of 1,028 data trials per subject. In addition, each subject began the session with six practice blocks of trials (768 trials). The experiment took about $1.5 \mathrm{~h}$, including rest periods, in a single experimental session.

Subjects. Sixteen subjects, ages 14 to 22 years, were recruited through a newspaper advertisement. They were paid $\$ 3$ for their participation. All subjects had normal or corrected-to-normal vision.

\section{Results and Discussion}

An analysis of percentage correct showed that successive presentation led to significantly better performance than did simultaneous presentation $[86.1 \%$ vs. $82.0 \%$, $\mathrm{F}(1,14)=10.67, \mathrm{p}<.01]$. The type of presentation did not interact with any other variable (all Fs $<1.0$ ). The subjects were reliably better in the second repetition than in the first repetition $[83.3 \%$ vs. $84.9 \%, F(1,14)=$ $8.95, \mathrm{p}<.025]$. For successive presentation, there was no significant difference between ISIs $[\mathrm{F}(1,14)=1.42]$. The average percentages correct for ISIs of 20 and 70 msec were $88.3 \%$ and $83.9 \%$, respectively. The lack of reliable effect for ISI replicates the results of Eriksen and Spencer (1969), but not those of Hoffman (1978). Homogeneous noise led to significantly better performance than did heterogeneous noise $[85.8 \%$ vs. $82.4 \%$, $F(1,14)=15.76, p<.005]$. A test of the effect of target-noise confusability can be made using data from the homogeneous-noise condition. The eight different homogeneous-noise items differed in performance $[F(7,98)=9.67, p<.001]$. The difference between the homogeneous noise items did not interact with the type of presentation $(F<1.0)$

These results support the theory that there are limitations in perceptual processing capacity. Furthermore, the difference between simultaneous and successive presentation was as great for the second half of the experiment as for the first half, which indicated that, within the limits of the experiment, perceptual processing capacity was unaffected by practice. Schneider and Shiffrin (1977) found large differences between CM and VM paradigms in far fewer trials than in the present study. It therefore seems unlikely that our results are a product of insufficient practice.

\section{EXPERIMENT 2}

\section{Method}

Experiment 2 tested the possibility that the advantage for successive presentation that was found in our Experiment 1 was due to more lateral masking with simultaneously presented items than with successively presented items. For half the subjects the display items were $1 \mathrm{deg}$ of visual angle apart (center to center), and for half the subjects the display items were 2 deg apart. If the difference between simultaneous and successive presentation in Experiment 1 resulted from greater lateral masking with simultaneous than with successive presentation, one would expect this difference to be greater when display items were close together. An ISI of $70 \mathrm{msec}$ was used. Experiment 2 was identical to Experiment 1 in all other details. The subjects, eight in each group, were drawn from the same subject pool as in Experiment 1, and five subjects in each group had participated in Experiment 1.

\section{Results}

Performance with successive presentation was better than performance with simultaneous presentation 
$[79.1 \%$ vs. $75.0 \%, F(1,14)=14.86, p<.01]$. Performance by the group with display items 1 deg apart was significantly better than performance by the group with display items 2 deg apart $[85.2 \%$ vs. $68.8 \%, F(1,14)=$ $21.7, \mathrm{p}<.01]$. The spacing of items did not significantly interact with the type of presentation $(F<1.0)$. There was a $4.2 \%$ advantage for successive presentation for the display items 1 deg apart and a $4.0 \%$ advantage with the display items $2 \mathrm{deg}$ apart. The worse performance with $2 \mathrm{deg}$ of separation suggests that the more peripheral placement of items at $2 \mathrm{deg}$ than at $1 \mathrm{deg}$ overcame any benefit resulting from the reduction of crowding in the 2-deg spacing. The overall mean difference in the two cases does not, however, affect our conclusion that the poorer performance in simultaneous presentation does not result from lateral interference.

Repetition was not significant $(F<1.0)$. The reason for the lack of a practice effect in this experiment may have been that most of the subjects in this experiment had participated in Experiment 1 and had probably reached an asymptotic level of performance. Arrays with homogeneous noise produced significantly better performance than those with heterogeneous noise $[78.6 \%$ vs. $75.5 \%, F(1,14)=38.4, p<.01]$.

\section{EXPERIMENT 3}

\section{Method}

Experiments 1 and 2 demonstrated that successive presentation leads to better performance than does simultaneous presentation in a forced-choice task when subjects are consistently searching for the same two targets. The purpose of this experiment was to extend these findings to a yes-no search task and thus to replicate the results of Duncan (1980b). The procedure was the same as that used in Experiment 1 with Group 2 (an ISI of $70 \mathrm{msec}$, items $1 \mathrm{deg}$ of visual angle apart), except that on each trial the subjects had only to indicate whether or not the letter $\mathrm{F}$ appeared. The subjects were told that on $50 \%$ of the trials the F would appear. Eight subjects were recruited as before; four of these had participated in Experiment 1 or Experiment 2 .

\section{Results}

Measured with $\mathrm{d}^{\prime}$, successive presentation was reliably better than simultaneous presentation $[F(1,6)=6.86$, $\mathrm{p}<.05]$. The mean $\mathrm{d}^{\prime}$ for simultaneous presentation was 1.27 , and for successive presentation it was 1.59 . Hit rates for simultaneous and successive presentation were .75 and .81 , respectively. The corresponding falsealarm rates were .28 and .30 . Performance for homogeneous noise was reliably better than that for heterogeneous noise $[\mathrm{F}(1,6)=42.97, \mathrm{p}<.001]$. The $\mathrm{d}^{\prime}$ scores for homogeneous and heterogeneous noise were 1.79 and 1.08 , respectively. Although the subjects were slightly better on the second repetition than on the first repetition (1.37 vs. 1.49), this effect was not reliable $[F(1,6)=3.08, p<.1]$. Similarly, the group with previous experience performed slightly better than the group without previous experience (1.38 vs. 1.48), but this effect was also not reliable $[\mathrm{F}(1,6)<1.0]$. There were no significant interactions.

\section{EXPERIMENT 4}

\section{Method}

Experiment 4 determined whether the temporal onset order of the target in successive presentation affects performance. Several theories assume that items must wait in a buffer (a "queue") until a central processor can analyze them (Fisher, 1982; Hoffman, 1978). These theories predict worse performance on targets presented later in the sequence. Queuing theories also predict an interaction between ISI and temporal onset order of the target. The longer the ISI, the shorter the time a late-appearing item must remain in queue, because earlier items would have time to be processed during the ISI.

This experiment used a circular array of eight letters. The noise item was always the letter $\mathrm{E}$. The task was a two-alternative forced-choice task, as in Experiments 1 and 2. Within a block there were 128 trials, consisting of 8 target positions $\times 2$ targets (the letters $\mathrm{F}$ and $\mathrm{T}$ ) $\times 8$ onset orders for the target (with successive presentation). The type of presentation (simultaneous vs. successive), the ISI for successive presentation ( $20 \mathrm{vs.} 100 \mathrm{msec}$ ), and the distance between items (items were located in a circle of either 1- or 1.5-deg radius) were varied between blocks. Each of eight subjects received each block of trials just once, in a Latin square design.

\section{Results}

Successive presentation led to significantly better performance than did simultaneous presentation $[83.2 \%$ vs. $78 \%, F(1,7)=10.54, p<.025]$. The type of presentation did not significantly interact with any other variable. Performance with items on a circle with a radius that subtended $1 \mathrm{deg}$ of visual angle was better than performance with items on a circle with a radius that subtended $1.5 \mathrm{deg}$ of visual angle [68\% vs. $73 \%$, $\mathrm{F}(1,7)=28.3, \mathrm{p}<.005]$. For successive presentation, analysis of the eight target onset orders was not significant $(F<1.0)$. The percentages correct for targets presented as the first, second, seventh, and eighth items were $81.5 \%, 82.0 \%, 83.4 \%$, and $83.2 \%$, respectively. Furthermore, neither ISI $(\mathrm{F}<1.0)$ nor the interaction of ISI and target onset order were significant $[F(7,49)=$ 1.17]. The lack of an effect of target onset order and the lack of an interaction between onset order and ISI argue against a queuing theory. There were no other significant main effects or interactions.

\section{EXPERIMENT 5}

\section{Method}

The purpose of the final experiment was to see if capacity limits in a CM paradigm could be eliminated with very extensive practice. Three subjects were tested for $1.5 \mathrm{~h} /$ day for 7 consecutive days. On each day, data were collected over eight blocks of 128 trials, for a total of 7,168 trials. Blocks were presented in a different random order for each subject on each day. On the 1 st day, the subjects received 100 practice trials of simultaneous presentation and 100 practice trials of successive presentation. On the following days, the subjects received 15 practice trials of each type of presentation. In all other respects the experiment was the same as Experiment 4. 


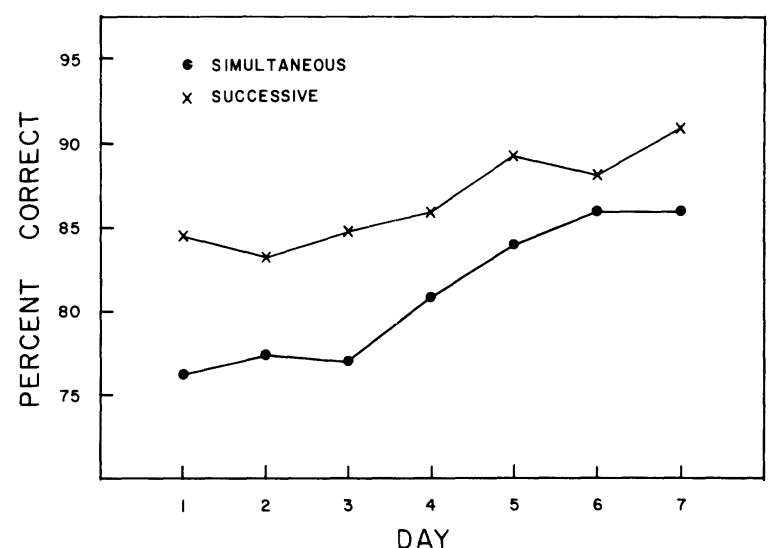

Figure 1. Detection accuracy as a function of practice in Experiment 5. On each day, data were collected over 1,024 trials.

\section{Results and Discussion}

Performance for arrays that subtended the smaller visual angle was better than that for arrays that subtended the larger visual angle $(85.8 \%$ vs. $82.2 \%)$. This factor did not significantly interact with any other factor. For successive displays, there was no effect of ISI $(F<1.0)$. As shown in Figure 1, performance improved with day $[\mathrm{F}(6,12)=5.45, \mathrm{p}<.01]$, and successive presentation was better than simultaneous presentation $[86.5 \%$ vs. $81.3 \%, F(1,2)=40.25, \mathrm{p}<.025]$. Not shown is the fact that each subject performed better on successive presentation on each day. Finally, the type of presentation interacted with day $[F(6,12)=$ $4.17, \mathrm{p}<.025]$. A simple main-effects test showed that performance with both simultaneous and successive presentation improved reliably over the week $[\operatorname{Fs}(6,12)=$ 3.02 and 7.02 for successive and simultaneous presentation, respectively]. These results indicated, for the first time in the present research, that the effect of capacity limits might have been getting smaller with practice. However, the interaction between type of presentation and practice might simply have been a result of a ceiling effect, which would have limited successive performance more than simultaneous performance. Regardless of whether capacity limits were truly being expanded, after over 7,000 trials, subjects still could process one letter at a time better than eight letters at a time.

\section{GENERAL DISCUSSION}

In five experiments in which subjects consistently searched for the same targets, there was a significant advantage for successive over simultaneous presentation. Our results, showing an advantage for successive presentation in a CM paradigm that cannot be attributed to nonperceptual factors, add to a growing body of literature that indicates capacity limits in recognition. As discussed earlier, Duncan (1980b) and Hoffman (1978, 1979) have found an advantage for successive presentation. Fisher (1982) modeled the data from several detection experiments and showed that as long as the display size of the stimulus array is under about five items, an unlimited-capacity theory can successfully model the data. However, as the number of items further increases, there are limits on processing. Shaw (1980, in press) showed that the display-size effect can exceed the maximum decrement in performance predicted by decision theories. Finally, Hoffman and Nelson (1981) showed evidence for spatial selectivity in letter recognition that cannot be easily accounted for by unlimited processing capacity in the recognition of briefly presented letters. An implication of demonstrating capacity limits in visual recognition is that, as in other areas of cognitive psychology, we may be able to understand the process by studying its limits.

\section{REFERENCES}

Duncan, J. The demonstration of capacity limitations. Cognitive Psychology, 1980, 12, 75-96. (a)

Duncan, $\mathbf{J}$. The locus of interference in the perception of simultaneous stimuli. Psychological Review, 1980, 87, 272-300. (b)

Eriksen, C. W., \& Spencer, T. Rate of information processing in visual perception: Some results and methodological considerations. Journal of Experimental Psychology Monographs, 1969, 79(No. 2, Part 2).

Fisher, D. L. Limited channel models of automatic detection: Capacity and scanning in visual search. Psychological Review, 1982, 89, 662-692.

Hoffman, J. E. Search through a sequentially presented visual display. Perception \& Psychophysics, 1978, 23, 1-11.

Hoffman, J. E. A two-stage model of visual search. Perception \& Psychophysics, 1979, 25, 319-327.

Hoffman, J. E., \& Nelson, B. Spatial selectivity in visual search. Perception \& Psychophysics, 1981, 30, 283-290.

Schneider, W., \& Shiffrin, R. M. Controlled and automatic human information processing: I. Detection, search, and attention. Psychological Review, 1977, 84, 1-66.

ShAw, M. L. Identifying attentional and decision-making components in information processing. In $\mathbf{R}$. S. Nickenson (Ed.), Attention and performance VIII. Hillsdale, N.J: Erlbaum, 1980.

Shaw, M. L. Attending to multiple sources of information: I. The integration of information in decision making. Cognitive Psychology, 1982, 14, 353-409.

Shaw, M. L. Consequences for coding variability of the spatial distribution of attention: A fundamental difference between pattern and luminance detection. In Attention and performance $X$, in press.

Shiffrin, R. M., \& Gardner, G. T. Visual processing capacity and attentional control. Journal of Experimental Psychology, 1972, 93, 72-82.

Spoehr, K. T., \& Lehmkuhle, S. W. Visual information processing. San Francisco: Freeman, 1982.

(Manuscript received for publication December 27, 1982.) 